\title{
A ANSIEDADE NO PROCESSO DE ENSINO/APRENDIZAGEM DE ESPANHOL COMO LÍNGUA ESTRANGEIRA E A SUA RELAÇÃO COM O CONTATO LINGUISTICO EM CONTEXTOS DE IMERSÃO
}

\author{
LA ANSIEDAD EN EL PROCESO DE ENSEÑANZA/APRENDIZAJE DE \\ ESPAÑOL LENGUA EXTRANJERA Y SU RELACIÓN CON EL CONTACTO \\ LINGÜÍSTICO EN CONTEXTO DE INMERSIÓN
}

\begin{abstract}
THE ANXIETY IN THE TEACHING / LEARNING PROCESS OF SPANISH FOREIGN LANGUAGE AND ITS RELATIONSHIP WITH THE LINGUISTIC CONTACT IN THE CONTEXT OF ENGLISH IMMERSION
\end{abstract}

RESUMO: O processo de ensino/aprendizagem de uma língua estrangeira depende de fatores externos como o contexto social ou sociocultural e, também, da influência dos fatores internos, como a motivação, os estilos cognitivos ou a ansiedade. Vários estudos apontam a ansiedade idiomática como o fator afetivo que obstaculiza com maior força o processo de aprendizado de uma língua estrangeira (Horwitz, Horwitz e Cope, 1986). Por esta razão, a finalidade deste artigo, é expor os resultados de uma investigação realizada com estudantes universitários americanos de língua espanhola em um contexto de imersão da cidade de Valencia- Espanha, para comparar se o contato linguístico frequente utilizando a língua meta exerce alguma influência na ansiedade idiomática, comparando os seus níveis de ansiedade ao princípio e ao final da estancia.

PALAVRAS-CHAVE: Ensino/aprendizagem. Espanhol língua estrangeira. Ansiedade idiomática. Contato linguístico. Contexto de imersão.

RESUMEN: El proceso de enseñanza/aprendizaje de una lengua extranjera depende de la influencia de factores externos como el contexto social o sociocultural y, también, de la influencia de factores internos como pueden ser la motivación, los estilos cognitivos o la ansiedad. Varios estudios señalan que la ansiedad idiomática es el factor afectivo que obstaculiza con mayor fuerza el proceso de adquisición de una lengua extranjera (Horwitz, Horwitz y Cope, 1986). La finalidad de este artículo es exponer los resultados de una investigación realizada con estudiantes universitarios estadounidenses de lengua española en un contexto de inmersión en la ciudad de Valencia, España, para comparar si el contacto lingüístico frecuente en la lengua meta ejerce alguna influencia en la ansiedad idiomática comparando los niveles de ansiedad al principio y al final de la estancia.

PALABRAS CLAVE: Enseñanza/aprendizaje. Español lengua extranjera. Ansiedad idiomática. Contacto lingüístico. Contexto de inmersión.

1 Universidade Federal de Pernambuco (UFPE), Recife - PE - Brasil. Profesora adjunta en el Departamento de Letras Español. ORCID: https:/orcid.org/0000-0002-5729-8755. E-mail: dayane_cordeiro@hotmail.com 
ABSTRACT: The teaching / learning process of a foreign language depends on the influence of external factors such as the social or sociocultural context, and also on the influence of internal factors such as motivation, cognitive styles or anxiety. Several studies indicate that language anxiety is the emotional factor that hinders the acquisition of foreign languages with greater force (Horwitz, Horwitz and Cope, 1986). The purpose of this article is to present the results of a research done on american university students American university students in Spanish in a inmersion contexto in the city of Valencia - Spain to compare if the frequent linguistic contact in the target language exerts some influence in the language anxiety by comparison of the anxiety levels at the beginning the and of the stay.

KEYWORDS: Teaching / learning. Spanish foreign language. Idiomatic anxiety Linguistic contact. Immersion context.

\section{Introducción}

Entre las emociones negativas, la ansiedad ha sido la emoción más estudiada tanto en la investigación educativa (FRENZEL ET AL., 2007) como en la adquisición de lenguas extranjeras (LE) ${ }^{2}$ (DEWAELE; MACINTYRE, 2014), debido a su impacto negativo a nivel afectivo y cognitivo durante el proceso de aprendizaje. Los estudios sobre el impacto de la ansiedad en el aprendizaje de una LE demuestran que este contexto específico genera más ansiedad que el aprendizaje de cualquier otra asignatura (RUBIO, 2004, p. 17).

El contacto lingüístico desvela la identidad del hablante al emplear el lenguaje para alcanzar sus objetivos comunicativos, y, por ello, podemos afirmar que la dimensión personal en inherente al lenguaje tanto en lengua materna, como en segundas lenguas o lenguas extranjeras. Los interlocutores en proceso de aprendizaje disponen de un repertorio lingüístico en proceso de desarrollo, que les impide, muchas veces, expresar todo su potencial comunicativo y esta situación puede producir ansiedad y frustración y llevar al alumno a evitar la comunicación (ARNOLD; FONSECA, 2004, p. 51).

Estas situaciones comunicativas insatisfactorias influyen de forma significativa en el autoconcepto del aprendiente como hablante competente y también en la valoración que otras personas harán de sus actos comunicativos (ARNOLD, 2011). Conocer las situaciones o prácticas de aula, que aumentan y disminuyen los niveles de ansiedad, así como reconocer las señales explícitas de la ansiedad al utilizar la lengua

${ }^{2}$ La referencia a "Lengua Extranjera" a partir de este punto se hará empleando la sigla LE. 
meta, ayuda a que el profesor disponga de técnicas y actividades capaces de reducir la influencia negativa de la ansiedad a favor de un aprendizaje más significativo (GREGERSEN et al., 2017)

\section{La ansiedad en el aprendizaje de lenguas extranjeras}

La ansiedad en el proceso de aprendizaje de una LE se ha definido como "un complejo distinto de autopercepciones, creencias, sentimientos y comportamientos que surgen de la singularidad del proceso de aprendizaje de idiomas "(HORWITZ; HORWITZ; COPE 1986, p. 128). Es decir, este tipo específico de ansiedad está vinculada a cualquier actividad realizada en el aula de LE, o empleando la lengua extranjera en contactos lingüísticos, como una "preocupación negativa" que dificulta el desarrollo de la competencia comunicativa (MACINTYRE, 1999, p. 27).

Los estudios realizados sobre la ansiedad idiomática demuestran que existe un vínculo entre los rasgos personales como, por ejemplo, las actitudes hacia la lengua meta, hacia el profesor, hacia los compañeros, hacia el aula, la autoestima y el autoconcepto; así como una gama de factores contextuales que repercuten en los niveles de ansiedad: el conocimiento de varios idiomas, la distancia o cercanía entre el idioma de origen y la lengua meta, el género, la edad, el ambiente del aula, etc. (DEWAELE, 2005). Es decir, existen múltiples factores que hacen con que la ansiedad en el aula de idiomas fluctúe durante todo el proceso, y, por ello, encontrar maneras de atenuar esta variable afectiva es una meta importante si buscamos ofrecer una enseñanza más humanística.

Con el objetivo de investigar con más detalle qué situaciones del aprendizaje de LE aumentan los niveles de ansiedad y de qué manera este factor afectivo repercute negativamente en el proceso de aprendizaje de una LE, Horwitz, Horwitz y Cope (1986) diseñan un instrumento completo capaz de medirla en diferentes situaciones de aula o de uso de la lengua. Este instrumento se conoce con la sigla FLCAS (Foreign Language Classroom Anxiety Escale) o escala que mide la ansiedad en contexto de adquisición de una LE.

La escala surge a partir de una investigación empírica realizada con un grupo de estudiantes que tenían dificultades en el aprendizaje de idiomas. Sobre la base de esta experiencia, reportada por los estudiantes de esta investigación, Horwitz et al. (1986) idearon una escala Likert compuesta por 33 ítems que se califican en una escala de 
cinco puntos que van de mayor a menor grado de acuerdo con lo preguntado. Los resultados indican que los niveles de ansiedad en una lengua extranjera oscilan y son más significativos en algunas etapas del proceso de aprendizaje de un nuevo idioma, y refuerzan que FLCAS es un instrumento de medida fiable ${ }^{3}$ para estos niveles, relacionándolos con tres tipos de situaciones específicas que desencadenan mayores niveles de ansiedad: la aprensión comunicativa, el miedo a la evaluación negativa y la ansiedad de examen.

Esta investigación se centra en el análisis de la aprensión comunicativa y su relación con el contacto lingüístico en contextos de inmersión. La aprensión comunicativa surge ante el temor de tener que realizar un acto comunicativo, principalmente durante el desarrollo de las actividades orales de clase, o en situaciones interactivas más espontáneas o con altos niveles de inmediatez, como responder a las preguntas del profesor, interactuar con él, con los compañeros, o con cualquier hablante nativo o competente en la LE. La aprensión comunicativa se asocia a varios factores de índole personal, que parten de "la autoestima y la autopercepción de la competencia oral personal, una baja tolerancia a la ambigüedad, el temor a cometer errores, la relación con los hablantes con los que el alumno puede emplear esta lengua" (GREGERSEN; MACINTYRE, 2014, p. 3).

Los hallazgos sobre la ansiedad y el rendimiento del lenguaje demuestran que la ansiedad obstaculiza el proceso de aprendizaje de varias maneras y en situaciones específicas (HORWITZ; HORWITZ; COPE, 1986; YOUNG, 1990; RUBIO, 2004). Los síntomas intelectuales o cognitivos de la ansiedad afectan al funcionamiento mental, debido a un estado subjetivo de tensión. Esta perturbación en las funciones psíquicas repercute en la atención, que se vuelve más dispersa, la memoria no responde bien a los estímulos y el pensamiento se lentifica (EYSENCK, 1979). Es decir, los síntomas intelectuales o cognitivos abarcan lo relativo al conocimiento: sensación, percepción, memoria, pensamientos, ideas, juicios, raciocinios y aprendizaje (RUBIO, 2004, p. 56) y son los más frecuentes en el contexto de enseñanza/aprendizaje de una LE.

Frente a lo expuesto, consideramos relevante comparar en qué medida la aprensión comunicativa está influenciada por el contacto lingüístico, y, en este sentido, cuáles son las situaciones comunicativas que generan mayores o menores niveles de ansiedad empleando la lengua meta.

${ }^{3}$ El coeficiente de consistencia interna del instrumento fue El alfa de Cronbach $=0,896(\mathrm{~m}=98,0$ y SD $=$ 15.17), que muestra una alta consistencia interna. 


\section{Metodología}

Para llevar a cabo este estudio, partimos de un contexto de inmersión en la ciudad de Valencia/España donde estudiantes universitarios estadounidenses, que realizan una estancia de mínimo cuarenta y máximo cien días, para profundizar sus conocimientos en español lengua extranjera en la Universitat de València. Además de la inmersión linguiística en un contexto de aprendizaje formal universitario, uno de los objetivos de este programa es proporcionar a los estudiantes vivencias culturales y personales que les acerque a la lengua meta, por ello, la mayor parte de los estudiantes se quedan alojados con una familia española.

Los datos se han recogido en dos cursos de español como lengua extranjera de nivel intermedio $\left(\mathrm{B} 1 / \mathrm{B} 1+/ \mathrm{B} 1++{ }^{4}\right)$. La muestra está compuesta por veinte alumnos universitarios, quince mujeres y cinco hombres, con edades entre diecinueve y veintiún años. El promedio de años de estudio de español de estos grupos es de dos años y siete meses y todos estudiaron español como mínimo un año en un contexto de enseñanza/aprendizaje universitario en su país de origen. Cabe destacar que, para dieciocho de los veinte alumnos, esta fue su primera experiencia de inmersión y, para dos alumnos, esta fue su segunda experiencia. Finalmente, de los veinte alumnos, trece afirman tener contacto habitual con el español por la situación de bilingüismo existente en muchas regiones de Estados Unidos.

Para comparar sus niveles de aprensión comunicativa antes y después de la estancia, elegimos utilizar la escala FLCAS ${ }^{5}$. La escala contiene 33 ítems que poseen una relación temática que se organizan en cinco subtemas relacionados a la ansiedad en el aprendizaje de una lengua extranjera. Para medir el nivel de ansiedad en cada uno de los ítems se emplea la escala Likert compuesta por 5 puntos que van desde "muy de acuerdo" a "totalmente en desacuerdo". Se suman los puntos de todas las categorías y cuánto mayor es la puntuación obtenida, mayor es el nivel de ansiedad.

Algunos autores consideran que el mero hecho de completar la escala de ansiedad puede tener un efecto saludable en sí mismo, ya que el alumno puede

${ }^{4}$ Según el MARCO EUROPEO DE REFERENCIA PARA LAS LENGUAS (MCER, 2002).

${ }^{5}$ Utilizamos la escala en versión original, la única adaptación que hicimos fue contabilizar de forma distinta las preguntas marcadas con un *. Estas preguntas no miden el nivel de ansiedad del alumno, porque la respuesta afirmativa no representa una actitud ansiosa por parte de este. En este caso, cuando la opción elegida fue "muy de acuerdo" contabilizamos 1 punto de esta respuesta, y si el alumno contesta "estoy en desacuerdo" contabilizamos 4 puntos y "totalmente en desacuerdo" 5 puntos, y así sucesivamente. Consideramos esta adaptación imprescindible, para que la medida de ansiedad presentada en los resultados representase la ansiedad real de los estudiantes investigados, dado que cuanto mayor es la suma de los ítems, mayor es la ansiedad del estudiante. 
reflexionar sobre sus pensamientos y preocupaciones negativas con relación al aprendizaje del idioma, convirtiendo la influencia de los factores afectivos en algo natural dentro del proceso de aprendizaje de una LE (ORTEGA, 2000, p.7). Es decir, FLCAS no solo sirve como un instrumento de reflexión para los estudiantes sobre cómo se sentían al principio y al final de la estancia, sino también como una herramienta de reflexión interpersonal.

Finalmente utilizamos las entrevistas personales grabadas y $\operatorname{transcritas}^{6}$ como un recurso de recogida de datos para ampliar nuestro análisis, a partir de respuestas personales relacionadas con el proceso de aprendizaje y la experiencia de contacto lingüístico con la lengua meta en contexto de inmersión que nos ayudaran a triangular estos datos con los resultados de FLCAS.

\section{Análisis y discusión de datos}

Tomamos algunas medidas para garantizar la fiabilidad de los resultados que presentamos a continuación; en primer lugar, en lo que se refiere a las circunstancias externas, la escala fue administrada por la investigadora en las dos ocasiones. La escala que mide la ansiedad idiomática (FLCAS) fue aplicada en la primera y en la última semana del curso en versión original (en inglés) garantizando así que todos los alumnos comprendieran todas y cada una de las cuestiones planteadas. Los alumnos dispusieron de 20 minutos para contestar las preguntas del cuestionario.

Antes de contestar la escala por primera vez, hicimos un repaso sobre las emociones y los sentimientos en español y preguntamos a los alumnos qué emociones suelen experimentar en el aula de ELE, a continuación, pedimos que contestasen la escala de forma sincera, ya que el objetivo del estudio era ofrecer unas condiciones óptimas de aprendizaje durante el curso, y conocerlos mejor nos ayudaría a alcanzar este objetivo. En ningún momento informamos de que esta escala serviría para medir la ansiedad, evitando así que las respuestas de los alumnos fuesen condicionadas a las instrucciones ofrecidas previamente.

A continuación, presentamos los resultados del análisis de la ansiedad al principio y al final de la estancia:

6 Sistema de transcripción de conversaciones «Val.Es.Co» que se puede consultar en https://www.uv.es/valesco/sistema.pdf 
Imagen 1 - Niveles de ansiedad de los grupos de investigación antes y después de la estancia

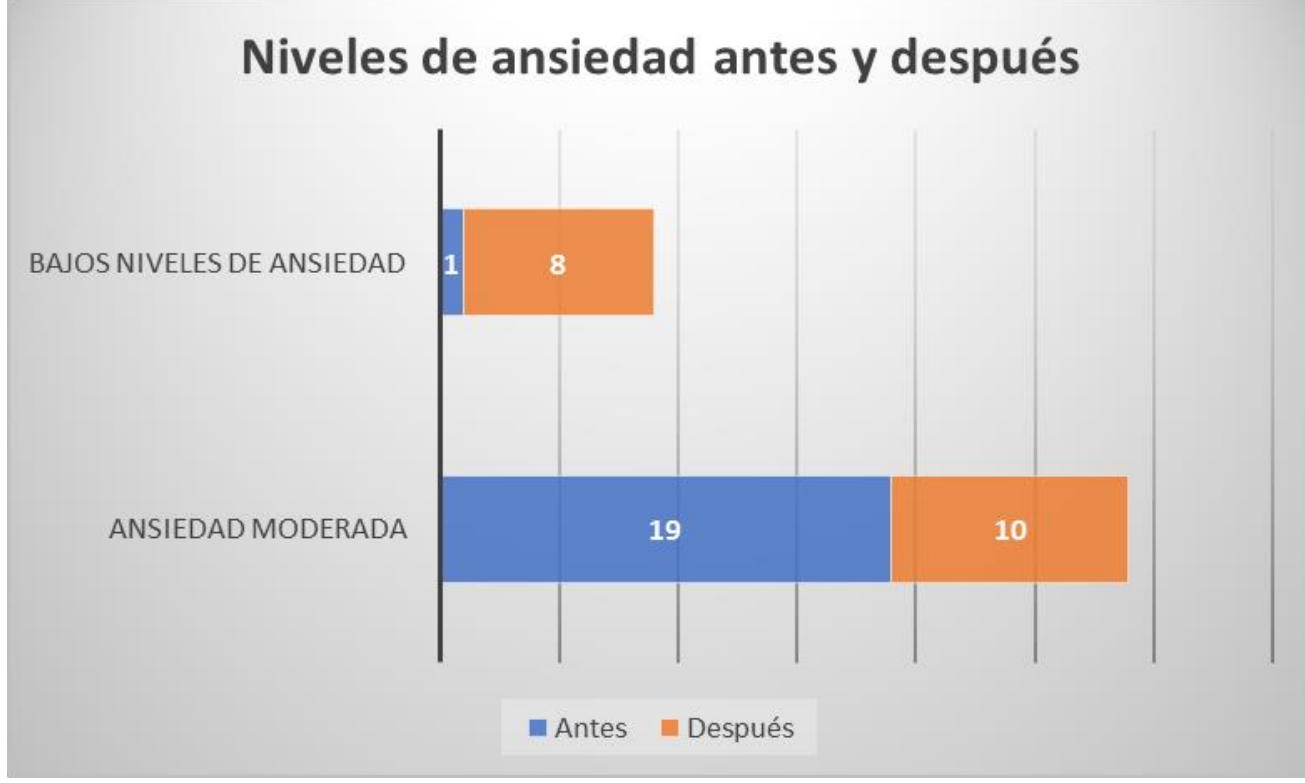

Los resultados del análisis de la primera escala revelan que en la muestra investigada no hay ningún participante con altos niveles de ansiedad idiomática. Prevalece la ansiedad moderada representada por diecinueve de los veinte alumnos: tres alumnos posen una ansiedad moderada alta, catorce alumnos una ansiedad moderada y dos alumnos una ansiedad moderada baja. Solamente una alumna posee una baja ansiedad a las situaciones del aula.

Los resultados de la escala al final de la investigación señalan que hubo una mejora cualitativa en la disminución de los niveles de ansiedad de los alumnos. Los niveles de ansiedad moderada y baja están más igualados que al principio del estudio. No obstante, el $50 \%$ de los alumnos sigue presentando una ansiedad moderada. En la segunda escala hay una alumna con una ansiedad moderada alta, siete alumnos presentan una ansiedad moderada, y cuatro alumnos una ansiedad moderada baja. El $40 \%$ de los alumnos presentan una baja ansiedad idiomática, lo que supone un resultado muy positivo: cinco alumnos presentan una ansiedad baja, y tres alumnos una ansiedad muy baja.

Cuatro alumnos mantuvieron los mismos niveles de ansiedad comparando el antes y el después: una alumna con bajos niveles de ansiedad, dos alumnas con ansiedad moderada, y una alumna con un nivel de ansiedad moderada alta. Consideramos este resultado parcialmente positivo, ya que, aunque no hubo una disminución de los niveles de ansiedad, que era lo deseado, tampoco hubo un aumento, por lo cual podemos intuir 
que el contacto lingüístico en un contexto de inmersión juntamente con el curso de español no tuvieron un impacto positivo ni negativo en sus niveles de ansiedad.

De igual forma, cabe reflexionar que aunque no existe un consenso sobre los aspectos positivos de la ansiedad en el aula, algunos estudios (HORWITZ, 1986; OXFORD; EHRMAN, 1991) destacan que la ansiedad en cuantidades moderadas no es perjudicial en el proceso de aprendizaje de una LE; el estado de alerta que los bajos niveles de ansiedad proporcionan puede ser útil para algunos alumnos en el desarrollo de tareas sencillas o en situaciones de competitividad en el aula, utilizando elementos de gamificación. Sin embargo, para las tareas que exigen un mayor esfuerzo cognitivo, o mayor inmediatez comunicativa, la ansiedad obstaculiza el proceso de aprendizaje.

En el siguiente epígrafe, presentamos los resultados sobre la ansiedad y su relación con algunas situaciones de uso de la lengua que pueden generar aprensión comunicativa según el análisis de las respuestas de FLCAS. Esta reflexión nos ayudará a comprender si el contacto lingüístico más frecuente en un contexto natural tuvo un impacto positivo en los niveles de ansiedad, así como conocer cuáles son las situaciones interactivas que generan mayores niveles de ansiedad en el grupo investigado.

\section{Las situaciones disparadoras de la aprensión comunicativa en el contacto lingüístico en contextos de inmersión}

Como destacamos en la metodología el instrumento utilizado para medir la ansiedad idiomática de los alumnos fue el cuestionario FLCAS, que está compuesto por 33 preguntas. Las relaciones temáticas entre los diferentes ítems de la escala permiten analizar cinco situaciones de aula que son propicias a generar ansiedad en el alumno:

- La ansiedad al expresarse.

- El temor a que se le corrija.

- Dificultades o falta de comprensión.

- Sentirse menos competente que los demás.

- Actitudes específicas hacia el aula de LE.

A continuación, se presentan los resultados de las cuestiones de la escala que tratan sobre la ansiedad al expresarse. Seleccionamos las respuestas de esta categoría porque se relaciona directamente con el tema central de este artículo, el contacto lingüístico en contextos de inmersión y su influencia en la aprensión comunicativa. 
Ocho ítems de la escala hacen referencia a la ansiedad al expresarse, las situaciones se relacionan con la percepción de seguridad versus inseguridad al expresarse verbalmente, por un lado, con los compañeros y con el profesor, y, por otro, con hablantes nativos de español. Asimismo, hay una cuestión sobre la importancia que los alumnos atribuyen a la percepción que los demás tienen de su competencia oral. A continuación, presentamos las preguntas ordenadas de mayor a menor grado de ansiedad según las respuestas de los alumnos comparando el antes y el después del plan de acción.

Tabla 1 - Ansiedad al expresarse de los grupos de investigación al principio y al final del curso

\section{La ansiedad y su relación con la expresión oral}

14. I would not be nervous speaking in Spanish with native speakers

9. I start to panic when I have to speak without preparation in the Spanish class.

1. I never feel quite sure of myself when I am speaking in my Spanish class.

24. I feel very self-conscious about speaking in Spanish in $30 \%$ $10 \%$ front of other students.

27. I get nervous and confused when I am speaking in my $30 \%$ $5 \%$ Spanish class.

20. I can feel my heart pounding when I'm going to be $30 \%$ $20 \%$ called on in the Spanish class.

32. I would probably feel comfortable around native $25 \%$

Spanish speakers.

18. I feel confident when I speak in the Spanish class

$10 \%$ $5 \%$

La comparación de las escalas al principio y al final del curso evidencia que las situaciones que generan mayores niveles de ansiedad se relacionan con la inseguridad al expresarse en español, tanto con hablantes nativos como en las interacciones en el aula. La inseguridad es una de las características relacionadas con la autoestima. Oxford (2000, p. 81) destaca que "la autoestima es vulnerable cuando el alumno se percibe a sí mismo como muy competente en su lengua propia y totalmente incapacitado o limitado respecto a la lengua meta". 
En este sentido, podríamos intuir que las situaciones que generan mayores niveles de ansiedad ocurren porque el alumno necesita comunicarse con un sistema lingüístico que no domina totalmente. Esta hipótesis nos llevaría a plantear que a medida que el alumno avanza en el proceso de aprendizaje, la ansiedad disminuiría. No obstante, MacIntyre y Gardner (1994b) consideran que a medida que el alumno progresa en el aprendizaje de una lengua extranjera, se enfrenta a una mayor cantidad de situaciones que pueden ser amenazantes porque exigen una mayor competencia lingüística. Esta afirmación permite intuir que las situaciones disparadoras de aprensión comunicativa no se relacionan solamente con la competencia lingüística, sino también con características personales y contextuales.

Comparando el antes y el después de la investigación, los resultados indican que hubo una reducción de la ansiedad a las situaciones de expresión oral de un 19\%. La triangulación de los datos nos permite atribuir esta mejora, por un lado, a las actividades y a las prácticas que desarrollamos en el aula y, por otro, al contexto de inmersión. Presentamos los datos que nos permiten relacionar mejores niveles de ansiedad con contexto de inmersión.

Tal y como se presenta en el cuadro anterior, la situación de expresión oral que genera mayor ansiedad según las respuestas de los alumnos es el contacto con hablantes nativos. El $45 \%$ de los alumnos afirman que se pondrían nerviosos frente a estas situaciones y al final del estudio solamente dos alumnos siguen con esta inseguridad. Este dato refuerza la hipótesis de que el contacto lingüístico más frecuente en situaciones cotidianas o en situaciones de habla real ayuda a reducir los niveles de ansiedad idiomática, porque favorece un uso de la lengua más comunicativo y contextualizado. A continuación, presentamos los testimonios de algunos alumnos sobre la importancia de este contacto con hablantes nativos para el desarrollo de las competencias orales:

A1: es muy interesante porque cuando yo am/ regresó a mi casal/puedo hablar con mi madre y mi familia española

A2: ... al principio nunca quería hablar yy/ y estabal/ oh no lo sé tenía vergüenza [risas] pero ahora es como ay valel likel no pasa nadall yo sé que cuando estoy hablando/ algunas veces la gramática no es correcto/l perooo cuando yo conocí amigos de España es cuando yo aprendí 
muchísimo más// no es solamente la lengua que practicamos en clasel es la lengua de la gentel la lengua más pura [risas]

Entrevista final A2-4

A5: ... tenía la oportunidad o tuve la oportunidad de usar la gramática cada día en conversación normal/ yy eso me ayudó muchísimo porqueel en los Estados Unidos es que podemos entender o podemos en/am/ aprender las lecciones yy/ el sistema de gramática lo que sea/ pero no tenemos la posibilidad/am/de interactuar/atulizarlo...

Entrevista final, A5-4

A6: am/ me gusta estudiar aquí porquee es inmersión/ es importante para mí porqueel/am/ es fácil aprender lengua en clase peroo/ vive lam/ la lengua es más interesantel es activo...

Entrevista final A6-1

A12: ... con mi familia solamento habloo en español con ellos y lam/ también las interacciones en la ciudad/cuando hablo con los trabajadores o también mis profesores oo lam/ también amigos españoles en los intercambios/ am/ todas las situaciones dan la oportunidad de mejorar mi español...

Entrevista final A12-4

No obstante, la siguiente pregunta, (n. $\left.{ }^{\circ} 32\right)$ que también enfatiza el contacto con nativos, presenta unos resultados contradictorios con relación a la pregunta anterior. Al principio de la investigación, en el 25\% de los casos, los alumnos estaban en desacuerdo con la afirmación de que se sentirían cómodos entre hablantes nativos de español, y al final del estudio el $35 \%$ de los alumnos afirman que no se sentirían cómodos. Estos datos permiten intuir que el contacto diario con hablantes nativos les hizo superar en cierta medida la ansiedad, pero no todos los alumnos se sienten cómodos en este tipo de contexto. Si contrastamos los resultados de otras categorías de análisis de FLCAS con las entrevistas, podemos sospechar que este aumento surge por los problemas de comprensión en el contacto con los hablantes nativos que utilizan una variable dialectal con la cual los alumnos no tienen tanta cercanía.

A6: primero am/era difícil para mí porquelaa vocabulario en España es diferente que vocabulario en Estados Unidos, no usar a vosotros // or the lik/ pero ahora es más normal para mí/ me gusta

Entrevista final, A6-1 
Cabría investigar si estos resultados se relacionan con factores socioculturales o por dificultades lingüísticas que los investigados pueden enfrentarse en las situaciones de expresión e interacción oral con hablantes nativos.

Asimismo, una de las alumnas relata que la convivencia con nativos en su caso no fue del todo beneficiosa, ya que sus compañeros también querían practicar el inglés con ella y por eso sus oportunidades de actuación en lengua española fueron más limitadas frente a los compañeros que se alojaron con familias españolas.

A3: pienso que mejorar mi am/ mi grado de hablar/l pero también ahora mismo tengo miedo a hablar un pocol/porquee vivo con estudiantes españoles y los chicos dee/ mi residencia am/ son simpáticos perol/es diferentel no hablan mucho conmigo/ un poco síl pero un poco en inglés también...

Entrevista final A3-4

Otra alumna destaca que en algunas ciudades españolas hay más oportunidad de expresarse en español con hablantes nativos que en otras:

A13: me encanta Valencia más que otras ciudades en Es- paña porque en Barcelona y en Madrid hay muchas personas que no hablan conmigo en español / y aquí siempre en español...

Entrevista final, A13-4

Sobre la disminución de la ansiedad a las situaciones de expresión oral en el aula, se intuye que tanto las actividades de aula, como las situaciones de contacto lingüístico durante la estancia, aumentaron la confianza de los alumnos en su competencia lingüística y, por lo tanto, consiguieron reducir los niveles de ansiedad. Las evidencias registradas al final del estudio avalan que tan solo un $25 \%$ de los alumnos se ponen ansiosos al expresarse oralmente en clase sin preparación previa. El $20 \%$ de los alumnos sigue experimentando síntomas relacionados a la ansiedad, como el aceleramiento cardíaco cuando se solicita su intervención. Solamente dos alumnos afirman que no se sienten seguros de sí mismos cuando interactúan en clase, o que se preocupan por lo que los demás pensarán sobre su actuación en clase. Finalmente, un alumno afirma que el nerviosismo le impide expresarse adecuadamente en clase.

Para concluir este análisis, podemos afirmar que los alumnos en general se sienten seguros de su capacidad comunicativa en clase, tan solo dos alumnos estaban en desacuerdo con esta afirmación al final de la investigación, y un alumno mantuvo una percepción negativa sobre su capacidad comunicativa en clase. Estos datos nos permiten 
afirmar que al final de la investigación el 95\% de los alumnos investigados sienten confianza en su capacidad de expresarse en español. La triangulación de FLCAS con las respuestas de los alumnos en la entrevista final refuerza que esta confianza surge a partir de la creación de una atmósfera de aula positiva.

A3: ... la clase era muy relajantee y puedo hablar más porqueel es más trenq-trenq /// era más tranquila.

Entrevista final A3-3

A5: ...me encanta como fue familiar/ podemos hablar con los profesores más relejado/más tranquilo/am/ en los Estados Unidos noll yy me encantaban las lecciones// he aprendido un montón...

Entrevista final A5-2

A13: muy fácil todo/ en mis clases estaba relajada aquíl fue relajada en vez dee en los Estados Unidos/ am/ pienso que aquí mis clases son más relajadas/ no séé/ más estudiantes hablando conmigo en las clases...

Entrevista inicial A13- 3

Al mismo tiempo, la experiencia de vivir la lengua, en intercambios lingüísticos cotidianos, les hizo sentirse más seguros de sus interacciones y, por lo tanto, menos ansiosos porque disponían de un mayor número de prácticas reales empleando la lengua meta con sus compañeros, familias de acogida, profesores y también en situaciones cotidianas.

\section{Consideraciones finales}

El objetivo de este estudio fue comparar los niveles de ansiedad idiomática, más específicamente, los niveles de aprensión comunicativa, de estudiantes estadounidenses de español como lengua extranjera al principio y al final de su estancia de estudios en la ciudad de Valencia. Los resultados de este análisis, tal y como se describen, nos permiten afirmar que existen tres variables principales que influyen de manera positiva en la reducción de la ansiedad en intercambios orales: un clima de aula afectivo, la importancia de trabajar con actividades variadas en el aula y la oportunidad de vivir la lengua en situaciones cotidianas. 
Según el testimonio de los alumnos investigados, lo que les ayudó a superar las dificultades y comunicarse mejor en la lengua meta, en primer lugar, fue la creación de un clima afectivo en el aula. En la transcripción de las entrevistas finales aparecen reiteradas veces afirmaciones como: "en esta clase ellos no tengo estrés", "no tengo miedo a cometer errores", "al principio tenía miedo de comunicarme, pero ahora no me importa", "no soy fluente, pero tengo más confianza en mí español", etc. Estas afirmaciones nos hacen plantear que una práctica que conecta con las emociones de los alumnos puede ser efectiva para disminuir el efecto negativo que algunos factores personales pueden ejercer en el proceso de aprendizaje, como la ansiedad.

En segundo lugar, el análisis de los datos destaca la importancia de practicar la lengua en situaciones de habla variadas y que conectan con los intereses personales de los alumnos, y que permitan al alumno tener más autonomía. En este sentido, cabe destacar que las experiencias memorables con el uso de la lengua extranjera son importantes pero, también, hace falta trabajar diariamente para mantener la confianza de los alumnos frente a sus limitaciones, y, para ello, es necesario conocer las necesidades individuales de los alumnos, realizar prácticas variadas que conecten con sus formas preferidas de aprender y también controlar los tiempos de habla en el aula, ofreciendo al alumno la posibilidad de planificar sus respuestas o disponer de tiempo para contestar las preguntas propuestas.

Finalmente, el análisis destaca el contexto de inmersión como un predictivo de éxito, porque los contenidos trabajados en el aula pueden emplearse en su día a día. Asimismo, el contexto de vivencia y aprendizaje se convierte en un espacio común para compartir dudas o reinterpretar situaciones que les ayudan a disminuir la inhibición y aumentar su motivación hacia el aprendizaje de lengua española.

AGRADECIMIENTOS: Quisiera expresar mi gratitud a la agencia de fomento CAPES que permitió que este estudio pudiera realizarse y también a la Universitat de València que me acogió para desarrollar esta investigación.

\section{REFERENCIAS}

ARNOLD, J. Attention to Affect in Language Learning. Anglistik. International Journal of English Studies, v. 22, n. 1, p. 11-22, 2011. 
ARNOLD, J.; FONSECA MORA, M. C. Multiple intelligence theory and foreign language learning: A brain-based perspective. International Journal of English Studies, v. 4, p. 119-136, 2004.

DEWAELE, J. M. Investigating the Psychological and the Emotional Dimensions in Instructed Language Learning: Obstacles and Possibilities. The Modern Language Journal 89, p. 367-380, 2005.

DEWAELE, J. M.; MACINTYRE, P. D. The two faces of Janus? Anxiety and enjoyment in the foreign language classroom. Studies in Second Language Learning and Teaching, v. 4, p. 237-274, 2014.

EYSENCK, M. W. Anxiety, learning and memory: A reconceptualization. Journal of Research in Personality,v. 13. p. 363-385, 1979.

FRENZEL, A. C.; PEKRUN, R.; GOETZ, T. Girls and mathematics-A "hopeless" issue? A control-value approach to gender differences in emotions towards mathematics". European Journal of Psychology of Education, 22, p. 497-514, 2007.

GREGERSEN, T.; MACINTYRE, P. D.; MEZA, M. D. The Motion of Emotion: Idiodynamic Case Studies of Learners' Foreign Language Anxiety. The Modern Language Journal 98, p. 574-588, 2014.

GREGERSEN, T.; MACINTYRE, P. D.; OLSEN, T. Do you see What I Feel? An Idiodynamic Assessment of Expert and Peer's Reading of Nonverbal Language Anxiety Cues. En New Insights into Language Anxiety: Theory, Research and Educational Implications, edited by C. Gkonou, M. Daubney, and J.-M. Dewaele. Bristol: Multilingual Matters, p. 110-134, 2017.

HORWITZ, E. K.; HORWITZ, M. B.; COPE, J. Foreign language classroom anxiety. The Modern Language Journal 70.2, p. 125-132, 1986.

MACINTYRE, P. D. Language anxiety: A review of the research for language teachers. En Young, D. J. (Ed.). Affect in foreign language and second language teaching: A practical guide to creating a low-anxiety classroom atmosphere. Boston, MA: McGraw-Hill, p. 24-45, 1999.

MACINTYRE, P. D.; GARDNER, R. C. The subtle effects of language anxiety on cognitive processing in the second language. Language Learning, 44, p. 283-305, 1994b.

ORTEGA, L. El desarrollo de la competencia gramatical oral en una segunda lengua a través de la actuación lingüística: aproximaciones interaccionistas y cognitivas. En Muñoz, C. (ed.), Segundas lenguas: adquisición en el aula. Barcelona, Ed. Ariel, S.A., p. 197-229, 2000.

OXFORD, R. L. La ansiedad y el alumno de idiomas: nuevas ideas. En Arnold, J (ed) La dimensión afectiva en el aprendizaje de idiomas. Ed.1. Cambridge, University Press, p. 77-86, 2000. 
OXFORD, R. L.; EHRMAN, M.; LAVINE, R. Style wars: teacher-student style conflicts in the language classroom. En Magnan, S. S. (Ed.). Challenges in the 1990s for college foreign language programs. Boston, Heinle \& Heinle, p. 1-25, 1991.

RUBIO ALCALÁ, F. D. La ansiedad en el aprendizaje de idiomas. Huelva, Publicaciones Universidad de Huelva, 2004.

YOUNG, D. J. An investigation of student's perspectives on anxiety and speaking. Foreign Language Annals, 23, p. 539-553, 1990.

\section{Cómo citar este artículo}

CORDEIRO, Dayane Mónica. La ansiedad en el proceso de enseñanza/aprendizaje de español lengua extranjera y su relación com el contacto linguistico en contexto de inmersión. Rev. EntreLínguas, Araraquara, v. 5, n. esp. 1, p. 312-327, jul., 2019. EISSN: 2447-3529. DOI: 10.29051/el.v5iesp.1.12987

Submetido em: 01/06/2019

Aprovado em: 30/06/2019

Publicado em: 01/10/2019 